\title{
Influence of socioeconomic status on measures of skeletal muscle mass in children
}

\author{
H. D. McCarthy, C. Van Eeden and D. Samani-Radia \\ Public Health Nutrition Research Group, London Metropolitan University, Holloway Road, London N7 8DB
}

We have previously shown that children from a lower income background have a higher body mass index (BMI) and body fat level for their age compared with children from a higher income background ${ }^{(1)}$. This difference could, in part, underpin the greater prevalence of obesity-related morbidity in lower income groups. Conversely, skeletal muscle is an important protective tissue for cardiometabolic disease and we have recently developed child and adolescent references for this body component ${ }^{(2)}$. However, the impact of childhood socioeconomic status on this tissue mass is unknown. The objective of this study was to evaluate skeletal muscle mass and its balance with fat mass in children from two contrasting income groups.

A total of 2297 Caucasian children aged between 5 and 14 years from inner city London and from more affluent surrounding counties participated in the study. Income group was defined at the school level from the percentage of children eligible for free school meals. Height, weight and BMI were measured and fat mass (FM, kg), fat-free mass (FFM, $\mathrm{kg}$ ) and appendicular skeletal muscle mass (SMMa) (using segmental bioelectrical impedance analysis, Tanita BC418MA) were estimated. SMMa was expressed in kg, as percent of body weight and as percent of FFM. Muscle-to-fat ratio (MFR, SMMa (kg)/FM (kg)) was calculated. Variables were compared between income groups separately for boys and girls using unpaired tests within age ranges 5-7, 8-10 and 11-14 y.

Percent FFM was significantly lower in the lower income boys in all age groups $(p<0.001)$ and in the girls in the youngest age group $(\mathrm{p}<0.001)$. FFM $(\mathrm{kg})$ was significantly lower only in the oldest boys $(\mathrm{p}<0.01)$ and in the middle and oldest groups of girls $(\mathrm{p}<0.02)$ from the lower income background. SMMa $(\mathrm{kg})$ tended to be lower in the lower income children but reached significance for the oldest boys and girls $(p<0.05)$ and youngest girls $(p<0.005)$. When expressed as a percent of body weight, SMMa was significantly lower in the low income children in all age groups $(\mathrm{p}<0.003$ to $<0.001)$ except for the oldest girls $(\mathrm{P}=\mathrm{NS})$. When expressed at a percent of FFM, SMMa tended to be lower in the low income children but reached statistical significance only in the youngest boys and girls $(p<0.001)$ and in the oldest girls $(p<0.02)$. MFR was significantly lower across all age groups in both boys and girls ( $p$ ranging between 0.05-0.001) except in the oldest girls ( $p=$ NS).

This is the first study to demonstrate small but significant deficits in SMMa in children from a low income background compared with those of a higher socioeconomic status. The explanation for, and biological and health significance of these observations are unclear at this stage but could reflect income group differences in early nutrition and physical activity. Whether these deficits track into adulthood is unknown but if so, could translate into a greater risk for sarcopenia in individuals from a lower income background. Furthermore, given the clear income group effects on MFR, this could suggest a greater risk for individuals from a lower income background for sarcopenic obesity and cardiometabolic diseases as these conditions may reflect the balance between these two key tissues. Furthermore, the role of height differences between these two populations groups on SMMa needs to be evaluated.

1. Samani-Radia D \& McCarthy HD (2010) Int J Obesity 35, 128-133.

2. McCarthy HD, Samani-Radia D, Jebb SA et al. (2014) Ped Obesity 9, 249-259. 